\title{
NAC Family Transcription Factors in Tobacco and Their Potential Role in Regulating Leaf Senescence
}

\author{
Wei Li, Xiaoxu Li, Jiangtao Chao, Zenglin Zhang, Weifeng Wang and Yongfeng Guo* \\ Tobacco Research Institute, Chinese Academy of Agricultural Sciences, Qingdao, China
}

OPEN ACCESS

Edited by:

Munetaka Sugiyama,

The University of Tokyo, Japan

Reviewed by:

Mitsuhiro Aida,

Kumamoto University, Japan

Bernd Mueller-Roeber,

Universität Potsdam, Germany

*Correspondence:

Yongfeng Guo

guoyongfeng@caas.cn

Specialty section:

This article was submitted to Plant Development and EvoDevo,

a section of the journal

Frontiers in Plant Science

Received: 26 September 2018 Accepted: 07 December 2018

Published: 21 December 2018

Citation:

Li W, Li X, Chao J, Zhang Z, Wang $W$ and Guo Y (2018) NAC

Family Transcription Factors in Tobacco and Their Potential Role in Regulating Leaf Senescence.

Front. Plant Sci. 9:1900.

doi: 10.3389/fp/s.2018.01900
The NAC family is one of the largest families of plant-specific transcription factors (TFs) and NAC proteins play important regulatory roles in a variety of developmental and stress response processes in plants. Members of the NAC family TFs have been shown to be important regulators of leaf senescence in a number of plant species. Here we report the identification of the NAC family in tobacco (Nicotiana tabacum) and characterization of the potential role of some of the tobacco NAC TFs in regulating leaf senescence. A total of 154 NAC genes (NtNACs) were identified and clustered together with the Arabidopsis NAC family into fifteen groups (a-o). Transcriptome data analysis followed by qRT-PCR validation showed that the majority of the senescence-up-regulated NtNACs fall into subgroups NAC-b and f. A number of known senescence regulators from Arabidopsis also belong to these two subgroups. Among these senescence-up-regulated NtNACs, NtNAC080, a close homolog of AtNAP, is a positive regulator of leaf senescence. Overexpression of NtNAC080 caused early senescence in Arabidopsis leaves and NtNAC080 mutation induced by Cas9/gRNA in tobacco led to delayed leaf senescence.

Keywords: NAC family, leaf senescence, NtNAC080, bioinformatic analysis, transcriptome, tobacco

\section{INTRODUCTION}

Senescence is the final stage of leaf development and is critical for plants' life cycle. As an important mechanism of evolutionary fitness, nutrients are remobilized from senescing leaves to other parts of the plant such as developing seeds and young leaves through the senescence process. Leaf senescence is an active, genetically controlled and highly regulated process accompanied by differential expression of 1000s of genes, including those with regulatory functions (Lohman et al., 1994; Hajouj et al., 2000; van der Graaff et al., 2006; Lim et al., 2007; Balazadeh et al., 2008; Guo, 2013; Ali et al., 2018). At the transcriptional level, the drastic changes in gene expression during leaf senescence are driven by transcription factors (TFs), the activities of which are triggered by a combination of aging signals and environmental factors (Guo, 2013). Previous studies have identified a number of TFs that participated in the process of leaf senescence from a number of protein families such as NAC, WRKY, and MYB proteins. These TF proteins act as switches to cause differential gene expression by binding to specific cis-acting elements of target gene promoters, resulting in the activation and/or suppression of target genes during senescence (Gregersen and Holm, 2007; Balazadeh et al., 2008; Besseau et al., 2012; Zhang and Gan, 2012; Shah et al., 2013).

The plant-specific NAC TFs compose one of the largest plant TF families. A NAC protein possesses a well-conserved $\mathrm{N}$-terminal NAC domain ( $\sim 160$ amino acids) and a variable C-terminal transcription regulatory region (TRR) (Ooka et al., 2003; Olsen et al., 2005b). The DNA binding 
ability of NAC TFs is confined to the NAC domain which can be divided into five subdomains (A-E). The highly conserved Subdomains C and D may be responsible for DNA binding, whereas Subdomain A may be involved in dimerization. The divergent Subdomains B and E may be responsible for functional diversity of the NAC proteins. The highly divergent C-terminal TRR region of NAC TFs is suggested to confer the regulation diversity of transcriptional activation activities (Ernst et al., 2004; Olsen et al., 2005a; Balazadeh et al., 2011; Kjaersgaard et al., 2011; Christiansen and Gregersen, 2014; Lindemose et al., 2014). Besides, an $\alpha$-helical transmembrane (TM) motif present in some NAC proteins has a function to anchor onto either endoplasmic reticulum or plasma membranes (Seo et al., 2008), which may play important regulatory roles in abiotic stress responses (SangGyu et al., 2008; Li et al., 2016).

The NAC family proteins have been shown to participate in diverse biological processes, including development of root and shoot apical meristems (Takada et al., 2001; Vroemen et al., 2003), organogenesis (Yamaguchi et al., 2010), hormone signaling (Xie et al., 2002; Kim et al., 2006; Jensen et al., 2008), fruit ripening (Shan et al., 2012; Ríos et al., 2017; Tranbarger et al., 2017), response to biotic and abiotic stresses (Nakashima et al., 2007, 2012; Huang et al., 2015; Yan et al., 2017), fiber, and secondary cell wall development (Mitsuda et al., 2007; Mitsuda and Ohme-Takagi, 2008; Zhong et al., 2008; Li et al., 2012; Chai et al., 2015; Zhang et al., 2018). In addition, NAC proteins have been shown to play important roles in regulating leaf senescence in various plant species (Lee et al., 2012; Fan et al., 2015; Podzimska-Sroka et al., 2015; Takasaki et al., 2015; Pimenta et al., 2016; Mao et al., 2017). A total of twenty NAC genes were present in an ESTs library of Arabidopsis senescent leaf (Guo et al., 2004). In a gene expression profiling study, about 30 NAC genes showed altered expression during Arabidopsis leaf senescence (Breeze et al., 2011). Among the senescenceup-regulated NAC TF genes, AtNAP was first identified as a positive regulator of leaf senescence. Overexpression of AtNAP led to premature senescence, whereas leaf senescence of the atnap knockout mutant plants was significantly delayed (Guo and Gan, 2006). In addition to AtNAP, NAC TFs ORS1, ORE1, and ATAF1 have been shown to play a role in promoting senescence in Arabidopsis as positive regulators while JUB1 and VNI2 are negative regulators of senescence (Balazadeh et al., 2011; Yang et al., 2011; Wu et al., 2012; Rauf et al., 2013; Garapati et al., 2015).

The roles of NAC TFs in regulating senescence seem to be conserved across species. AtNAP homologous genes have so far been reported in a variety of plant species including rice, maize, wheat, soybean, kidney bean, peach, tomato, petunia, potato, poplar, tall fescue, and bamboo (Guo and Gan, 2014). Like AtNAP, the homologs of rice, kidney bean, bamboo, and cotton have been shown to be expressed in senescing leaves but not in non-senescing ones and, more importantly, the homologs function as AtNAP orthologs because they complemented the Arabidopsis nap null mutants (Guo and Gan, 2006; Chen et al., 2011; Fan et al., 2015). Furthermore, the AtNAP ortholog in rice, OsNAP, was shown to have the same regulatory role in rice leaf senescence (Liang et al., 2014). RNA silencing of the maize homolog, ZmNAP, caused a stay green phenotype in maize
(Zhang et al., 2012). Knocking down of the NAP ortholog in cotton, GhNAP, also caused a significant delay in leaf senescence (Fan et al., 2015).

The NAC family has been characterized in a number of plant species. These studies indicated that there are 105 NAC genes in Arabidopsis (Ooka et al., 2003), 151 in rice (Nuruzzaman et al., 2010), 163 in poplar (Hu et al., 2010), 74 in grape (Wang et al., 2013), 152 in soybean (Le et al., 2011), and 97 in Medicago truncatula (Ling et al., 2017). Using gene-space sequence reads (GSR) from methylation filtrated tobacco genomic DNA libraries, an earlier study on tobacco TFs reported 152 NAC domain genes in tobacco (Nicotiana tabacum L.) (Rushton et al., 2008). Taking advantage of the recently available high quality genome sequences of tobacco and its two ancestor species (N. sylvestris and N. tomentosiformis) (Gerstel, 1960; Sierro et al., 2013), in the current study we have identified the complete set of NAC proteins in tobacco, and have analyzed their gene structures, protein motifs and phylogeny. We also did a comprehensive analysis of the expression profile of all NtNAC genes using RNA-Seq data as well as quantitative real-time PCR validation. Moreover, potential roles of tobacco NAC members in regulating leaf senescence were predicted based on expression pattern and sequence homology, and function of one of the senescence-up-regulated NAC genes, NtNAC080, in leaf senescence was investigated.

\section{MATERIALS AND METHODS}

\section{Database Search and Sequence Retrieval of NAC Proteins From Nicotiana tabacum}

The tobacco genomic sequences were downloaded from SGN (Sol Genomics Network) ${ }^{1}$. The Hidden Markov Model (HMM) profile of NAC domain (PF02365) retrieved from Pfam $^{2}$ was used to conduct HMM search against the annotated protein database with an E-value cutoff of 1.0 using HMMER (v3.0) (Johnson et al., 2010). All non-redundant hits within expected values were collected and each newly identified hit was subsequently used as a query to perform BLASTP search against the annotated tobacco genome. The protein sequences obtained from the two abovedescribed approaches were combined and redundant entries were removed manually. The resulted non-redundant sequences were manually analyzed using InterPro to ensure presence of the NAC domain (Apweiler et al., 2001). Moreover, TMHMM Server ver.2.0 was used to predict the membrane-bound NtNAC members (Krogh et al., 2001).

\section{Gene Structure and Motif Analysis}

Exon-intron structures of tobacco NAC genes were analyzed and illustrated with the Gene Structure Display Server (GSDS) ${ }^{3}$ by comparison of gene CDS sequences with genomic DNA sequences obtained from the Sol Genomics Network. The program MEME version 4.3.0 was employed for the detection of conserved motifs

\footnotetext{
${ }^{1}$ http://solgenomics.net/

${ }^{2}$ http://pfam.sanger.ac.uk/

${ }^{3}$ http://gsds.cbi.pku.edu.cn/
} 
in tobacco NAC proteins ${ }^{4}$. MEME was run locally with the following parameters: distribution of motif occurrences, zero or one per sequence; maximum number of motifs, 10; optimum motif width, $\geq 6$ and $\leq 200$ (Bailey et al., 2015).

\section{Multiple Sequence Alignment and Phylogenetic Analysis}

Multiple sequence alignments of full-length tobacco NAC amino acid sequences were performed using the MAFFT program with the default parameters together with 105 Arabidopsis NAC protein sequences and two representative NAC proteins (StNAC010 and StNAC039) from potato (Solanum tuberosum L.) which have been reported to be in the Solanaceae-specific subfamily (TNACs) of the NAC family (Katoh and Standley, 2013). Sequences of Arabidopsis and potato NAC proteins were downloaded from TAIR $10^{5}$ and $\mathrm{SGN}^{6}$, respectively. Un-rooted phylogenetic trees were constructed via MEGA 6.06 using the Neighbor-Joining (NJ) method with the following parameters: Poisson model and bootstrap values of 1000 replicates. Pairwise deletion mode was employed to make sure that the divergent C-terminal domains could contribute to the topology of the phylogenetic trees (Tamura et al., 2013).

\section{Plant Materials and Growth Conditions}

Common tobacco (Nicotiana tabacum L. Cv. K326) plants were grown in the field. Middle leaves (Leaf No. 9-10 from the base of a tobacco plant) were collected at 15, 45, 65, 75 days after topping (DAT) (defined as YL, young leaf; ES, early senescing leaf; MS, mid-senescing leaf; LS, late senescing leaf, respectively) for analyzing gene expression during leaf senescence. All harvested leaves were wrapped in aluminum foil, immediately placed in liquid nitrogen and stored at $-80^{\circ} \mathrm{C}$ until used. Three biological replicates were analyzed for each time point. In gene functional analysis, The T1 plants of NtNAC080 CRISPR-Cas9 transgenic lines and wild-type plants (K326) were grown in the greenhouse under normal growth conditions.

Arabidopsis Col-0 and transgenic plants were grown in a plant growth chamber (Conviron, Canada) at $22^{\circ} \mathrm{C}$ with a relative humidity of $55 \%$ under long-day conditions ( $16 \mathrm{~h}$ light $/ 8 \mathrm{~h}$ dark) with white light illumination $\left(120 \mu \mathrm{mol}\right.$ photons $\left./ \mathrm{m}^{2} \mathrm{~s}\right)$.

\section{Gene Expression Profiling}

The data used for expression profiling of tobacco NAC genes were from the tobacco Illumina RNA-seq data generated in the Guo lab (Li et al., 2017) (archived by NCBI under the accession numbers: SRP102153). For RNA-Seq data of tobacco leaf senescence, RPKM values of the 154 NtNAC genes were retrieved and normalized. A heatmap was generated based on the $\log _{2}$ fold-change values at $25 / 35 / 45 / 55 / 65 / 75 D A T$ when compared with 15DAT and visualized with $R$ package ( $R$ Development Core Team, 2013). In addition, the data used for expression profiling of Arabidopsis NAC genes (ANAC genes) were retrieved from AtGenExpress leaf developmental data by

\footnotetext{
${ }^{4}$ http://meme.sdsc.edu

${ }^{5}$ http://www.arabidopsis.org/

${ }^{6} \mathrm{http}: / /$ solgenomics.net/
}

Schmid et al. (2005). Based on the previous Guo et al. analysis, we performed differential expression analysis of all the ANAC genes between the fully expanded leaves (young leaves) and senescing leaves (Guo and Gan, 2012). Genes that are upregulated twofold or more were designated as being senescence up-regulated ANACs.

\section{RNA Isolation and Quantitative Real-Time RT-PCR (qRT-PCR) Analysis}

Total RNA was extracted according to the method previously described (Li and Guo, 2018). Genomic DNA was eliminated from the RNA samples via DNase I (Takara bio inc., Otsu, Japan) treatment for $30 \mathrm{~min}$ at $37^{\circ} \mathrm{C}$. The quality and concentrations of RNA samples were determined using a Nanodrop 2000 spectrophotometer (Thermo Fisher Scientific, Wilmington, DE, United States). First-strand cDNAs were synthesized using the RevertAid H Minus First Strand cDNA Synthesis Kit (Thermo Scientific) with Oligo(dT) primers. qRT-PCR was performed on an ABI7500 Real-Time PCR System (Applied Biosystems, Foster City, CA, United States) with the reaction mixtures comprising $1 \mu \mathrm{L}$ template cDNA, $1 \mu \mathrm{L}$ each of forward and reverse primers $(0.3 \mu \mathrm{M}$ each) and $10 \mu \mathrm{L}$ FastStart Universal SYBR Green Master (Rox, Roche Applied Science). For tobacco, NtActin was used as an internal control. NtCP1 (SAG12 homolog in tobacco) and NtRBCS were used as senescence markers. For Arabidopsis, AtActin and AtSAG12 were used as the internal control and senescence marker, respectively. The relevant primers are given in Supplementary Table S1. All reactions were run in triplicate. The relative gene expression values were analyzed using the $2^{-\Delta \Delta \mathrm{Ct}}$ method.

\section{Overexpressing NtNAC080 in Arabidopsis}

The coding sequence of NtNAC080 was PCR amplified from K326 cDNA using the PrimeSTAR HS DNA polymerase (Takara Bio, Japan) and cloned into the binary vector pCHF3 (a modified PPZP212 vector) at the BamHI/SacI sites after the CaMV 35S promoter (Hajdukiewicz et al., 1994). Primers used in vector construction are listed in Supplementary Table S2. The construct was then used to transform Arabidopsis using the Agrobacterium floral dip method (Clough and Bent, 1998). Transgenic Arabidopsis plants were selected on kanamycin $(50 \mathrm{mg} / \mathrm{L})$ plates. Presence of the transgene was PCR confirmed using genomic DNA from the leaves of putative transformants. Abundance of the NtNAC080 transcript was estimated via qRTPCR using cDNA from senescing leaves of the transgenic plants.

\section{CRISPR-CAS9-Mediated Mutation Induction in Tobacco}

The DNA sequences of NtNAC080, NtNAC028, NtNAC083, and NtNAC110 are highly similar. To produce a gRNA specific to NtNAC080, an online software ${ }^{7}$ was used for synthesizing two DNA oligos, NtNAC080-gRNA1 and NtNAC080-gRNA2, The NtNAC080-sgRNA forward and reverse primers were denatured

${ }^{7}$ http://www.multicrispr.net/index.html 
by heating at $95^{\circ} \mathrm{C}$ for $3 \mathrm{~min}$ and annealed to form a double-stranded DNA. Thereafter, the double-stranded DNA was inserted into the $p O R E$-Cas9/gRNA vector at the BsaI site (Gao et al., 2015). Tobacco cultivar K326 was transformed with the resulting construct via Agrobacterium-mediated transformation as described earlier (Horsch et al., 1989). The shoots of putative transformants were selected in Murashige and Skoog (MS) medium containing $0.1 \mathrm{mg} / \mathrm{L}$ 1-naphthylacetic acid (NAA), $1 \mathrm{mg} / \mathrm{L}$ 6-dimethylaminopurine (6-BA), $50 \mathrm{mg} / \mathrm{L}$ kanamycin (Kan), and $500 \mathrm{mg} / \mathrm{L}$ cefotaxime sodium (Cef). Putative Kanresistant transformants were transferred to soil pots and grown under greenhouse conditions. The genomic DNA of transgenic tobacco plants from Kan selection were extracted using the DNeasy Plant Mini Kit (Qiagen, CA, United States). The DNA fragments containing the Cas9/gRNA target sequences were amplified by PCR using PrimeSTAR HS DNA polymerase (Takara Bio, Japan). After purification, the PCR product was cloned to the $p E A S Y$-Blunt Zero vector (Transgene, China) and the DNA from the single colonies were sequenced to detect the types of mutation. For the target gene edited tobacco plants, selfing was performed and the homozygous mutants were acquired from T1 transgenic plants. The relevant primers are given in Supplementary Table S2.

\section{Chlorophyll Extraction and Quantification}

Chlorophyll was extracted and quantified as described previously (He and Gan, 2002). Briefly, $10 \mathrm{mg}$ of freeze-dried leaf tissue was extracted with $1 \mathrm{~mL}$ of $95 \%$ ethanol in the dark for $24 \mathrm{~h}$ with agitation. The supernatant was quantified via spectrophotometric measurement at 649 and $665 \mathrm{~nm}$. Three biological replicates were used for each sample.

\section{RESULTS}

\section{Identification and Phylogenetic Analysis of NAC Family Members in Tobacco}

To identify NAC genes in tobacco, Hidden Markov Model (HMM) search was performed against the Sol Genomics Network database $^{8}$ using the Pfam NAC domain (PF02365) as query. Newly identified hits were used as queries to carry out BLASTP search until no further hit can be obtained. After manual removal of redundant hits, all the putative NAC proteins were further screened using the $\mathrm{Pfam}^{9}$ and the Interpro ${ }^{10}$ programs to ensure that each protein has a N-terminal NAC domain. Following this procedure, a total of 154 non-redundant NAC family proteins were identified in tobacco. The tobacco NAC genes were named NtNACs followed by Arabic numbers. The length of the NtNAC proteins ranges from 130 to 645 amino acids (aa) with an average of 330 aa. The detailed information of NAC family genes in tobacco, including accession numbers, protein lengths, protein sequences, conserved domains and

\footnotetext{
${ }^{8} \mathrm{http}: / /$ solgenomics.net/

${ }^{9}$ http://pfam.xfam.org/

${ }^{10}$ http://www.ebi.ac.uk/interpro/search/sequence-search
}

similarities to their Arabidopsis orthologs etc., is listed in Supplementary Tables S3, S4.

To study the evolutionary relationship between the tobacco NAC proteins and known NACs from other plant species, an unrooted phylogenetic tree was constructed from alignments of 154 NtNACs, 105 Arabidopsis ANACs and 2 representative potato NAC protein sequences (Figure 1). All the NAC proteins were divided into 15 distinct subgroups: NAC-a to NAC-o. The tobacco NAC members demonstrated an interspersed distribution in all the subgroups except the subgroup NAC-1, in which all members are from Arabidopsis. The subgroup NAC-o, on the other hand, contains no NAC from Arabidopsis. Further alignment analysis suggested that proteins in subgroup NACo shared sequence characteristics of TNACs, which have been reported to be the Solanaceae-specific NAC subfamily (Rushton et al., 2008). The two representative TNAC potato proteins, StNAC010 and StNAC039 (Singh et al., 2013), were also grouped in this subgroup, suggesting NAC-o being a Solanaceae-specific NAC subgroup.

\section{Gene Structure and Protein Motif Analysis of the NAC Gene Family in Tobacco}

To gain insights into the structural diversity of the NtNAC genes, the exon/intron structure of the coding sequences of individual NtNACs in tobacco was analyzed (Figure 2B). In general, members of the same subgroup share similar exon/intron structure and gene length. For example, the $N A C$ genes in subgroups NAC-a, NAC-b, NAC-d, NAC-e, and NAC-h contain one or two introns. The NAC-f members have two introns except $N t N A C 110$, which harbors three introns. In addition, 7 of the $N A C$ genes have no intron, all of which belong to subgroup NAC-o. The members in subgroups NAC-i and NAC-m on the other hand, have more variable gene structures. Among the 154 NtNACs, the shortest NtNAC gene is 474 bp (NtNAC104) long, whereas NtNAC068 is the longest NtNAC gene with a $10 \mathrm{~kb}$ genomic sequence.

Using the MEME program, 10 conserved motifs, named Motif 1-10, were identified in the NtNAC proteins, all located within the N-terminal region which are highly conserved for DNA-binding, as described previously (Singh et al., 2013; Wei et al., 2016; Figure 2C and Supplementary Figure S1). Motif 2/8, Motif 1/7, Motif 3, and Motif 4 comprise the NAC DNAbinding domain while other motifs are dispersed around the NAC domain. Similar motif organization was observed within the same subgroup or in closely related subgroups (Figures 1, 2A,C). For instance, only NAC members in the Solanaceae-specific subgroup NAC-o contain both Motifs 8 and 9. Subgroup NACf mainly contains Motifs 1, 2, 3, and 4. Most members of the subgroup NAC-m comprise Motif 3, 5, 8, and 10. A conserved nuclear localization signal (NLS) was identified within Motif 3 (Supplementary Figure S1). As expected, the C-terminal transcriptional regulation region (TRR) is variable.

A trans-membrane (TM) region with a predicted-helix was identified in the far C-terminal region of $13 \mathrm{NtNACs}$ proteins, which belonging to subgroups NAC-i, NAC-k and NAC-o. 


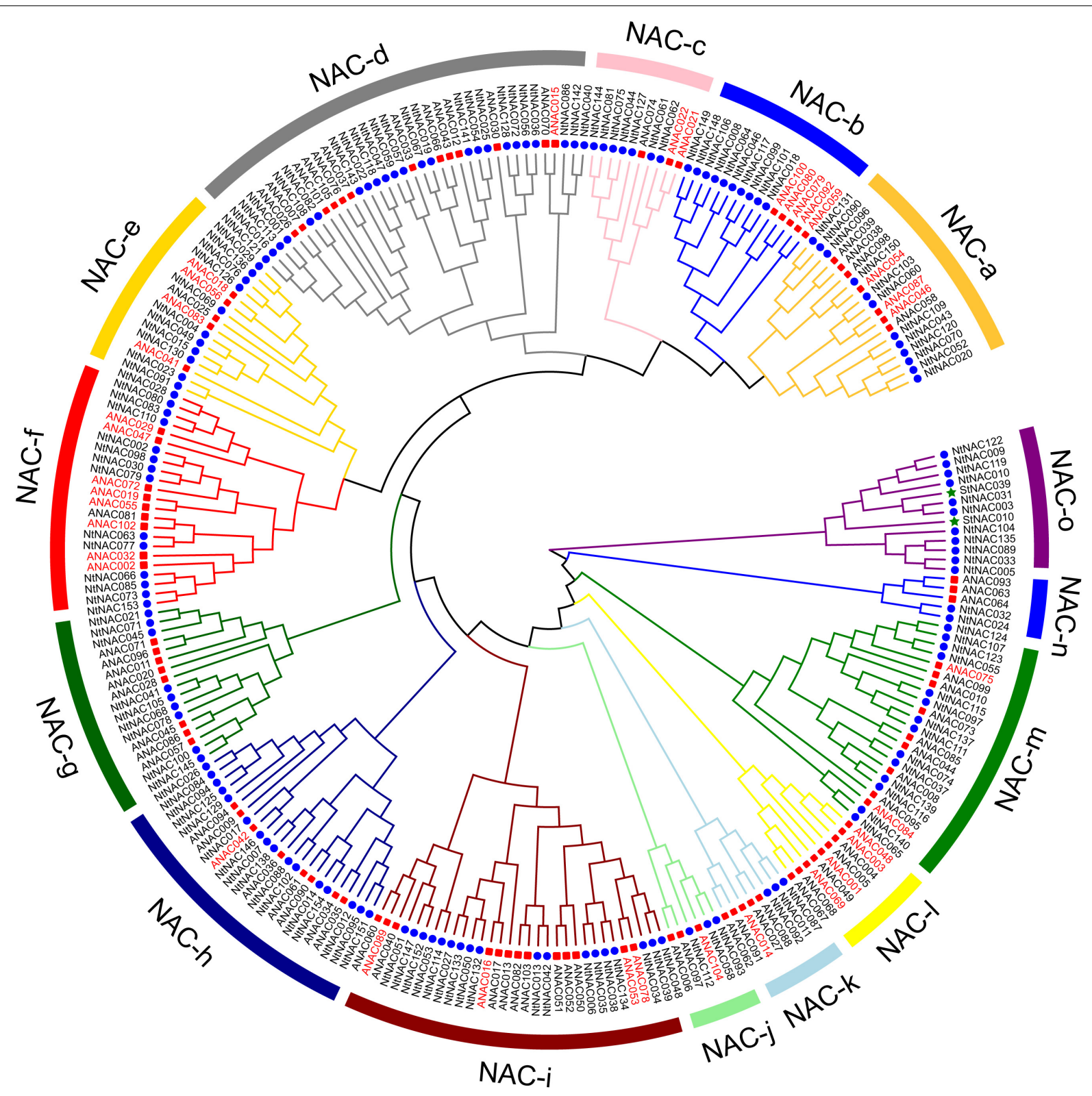

FIGURE 1 | Phylogenetic tree of NAC proteins of tobacco, Arabidopsis and potato. Multiple sequence alignments of NAC proteins were performed by MAFFT program with default parameters. The un-rooted phylogenetic tree was constructed by MEGA 6.06 with the Neighbor-Joining (NJ) methods using the following parameters: Poisson model and bootstrap values (1000 replicates). Pairwise deletion mode was employed to make sure that the divergent C-terminal domains could contribute to the topology of the phylogenetic tree. The tree was divided into 15 phylogenetic subgroups, designated as NAC-a to NAC-o. Members of tobacco, Arabidopsis and potato were denoted by blue spots, red squares and green asterisks. ANAC genes which were specifically up-regulated in senescing leaves are indicated by red color. Ratios of expression change for individual ANAC genes were presented in Supplementary Table S5.

Remarkably, subgroup NAC-i contains most of membranebound NAC members of tobacco and Arabidopsis, including 8 NtNACs and 7 ANACs (Supplementary Table S3). There are 18 membrane-associated NAC TFs in Arabidopsis, 5 in rice, 11 in soybean and 14 in potato (Kim et al., 2007, 2010; Singh et al., 2013). A number of Arabidopsis membrane-bound NAC proteins have been shown to be involved in biotic and abiotic stress responses (Kim et al., 2007, 2010; Lee et al., 2012).

\section{Prediction of NtNAC Genes Involved in Leaf Senescence Using Transcriptome Data}

NAC TFs play an important regulatory role in leaf senescence (Li and Guo, 2014). Transcriptomic studies have shown that approximately one third of the NAC genes were upregulated during leaf senescence in Arabidopsis, highlighting their importance in senescence regulation (Breeze et al., 2011). 


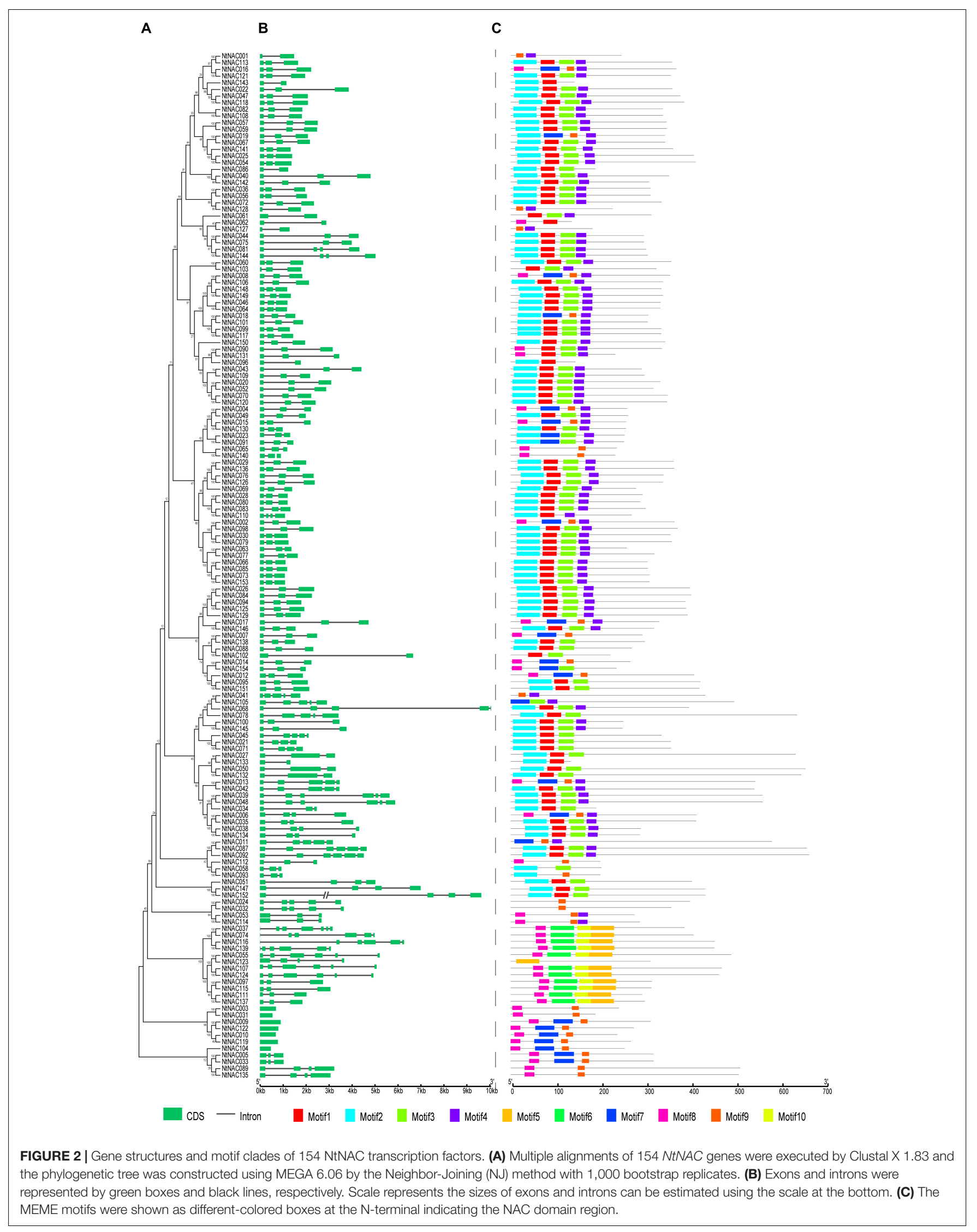


Using the publicly available gene-chip based data by Schmid et al. (2005), 36 ANAC genes which were up-regulated twofold or more during leaf senescence were identified (Supplementary Table S5). Interestingly, 13 of the senescence-up-regulated ANACs belong to the phylogenetic subgroups NAC-b and NAC-f, which totally contain 14 ANAC genes from Arabidopsis. Moreover, several ANAC TFs that have been previously identified as positive regulators of leaf senescence, including ANAC029/AtNAP, ANAC059/ORS1, ANAC092/ORE1/AtNAC2, ANAC002/ATAF1 (Guo and Gan, 2006; Balazadeh et al., 2011; Rauf et al., 2013; Garapati et al., 2015) were clustered together in the NAC-b and NAC-f subgroups. NAC genes with same functions showed a tendency to fall into the same subgroup (Fang et al., 2008; Hu et al., 2015; Wei et al., 2016). We thus predict that the NAC genes of NAC-b and NAC-f subgroups might play an important role in regulating leaf senescence.

To explore the patterns of tobacco NAC expression during leaf senescence, we performed a comprehensive analysis of NtNAC genes expression profiles at different stages of leaf senescence based on data retrieved from our earlier RNA-Seq study of tobacco leaf senescence ( $\mathrm{Li}$ et al., 2017). We were able to obtain transcripts data from most of the NtNACs (147 out of 154) from the dataset (Supplementary Table S6). As previously reported in Arabidopsis and other species (Breeze et al., 2011; Christiansen and Gregersen, 2014), the analysis of expression changes indicated significant transcriptional responses of the NtNAC genes during tobacco leaf senescence. Twenty four of the NtNACs were up-regulated fourfold or more at least at one time point from 25DAT to 75DAT compared with 15DAT (Figure 3 and Supplementary Table S6). Interestingly, 11 of these 24 genes were clustered in subgroups NAC-b and NAC-f, and are closely related to the Arabidopsis senescenceregulating NAC genes, including AtNAP/ANAC029, ANAC002, ANAC059, and ANAC092 (Figure 1). In addition, four other NtNAC genes in subgroups NAC-b and NAC-f, including NtNAC008, NtNAC018, NtNAC064, and NtNAC098, showed an up-regulation of 2-4-fold and most of the remaining genes in the two subgroups showed somewhat increased expression during senescence. NtNACs in subgroups NAC-b and NAC-f shared similar up-regulation patterns during senescence as their homologs in Arabidopsis, suggesting that these two subgroups may play significant roles in regulating leaf senescence and these regulatory roles might be conserved between species.

\section{Quantitative PCR Analysis of Selected NtNAC Genes During Tobacco Leaf Senescence}

To validate the result of RNA-Seq analysis, we analyzed the expression patterns of 15 selected NtNAC genes from the subgroups NAC-b and NAC-f during leaf senescence using quantitative RT-PCR (qRT-PCR). Data from 4 leaf developmental stages, including fully expanded, non-senescent leaf (YL), early senescent leaf (ES), middle senescent leaf(MS), and late senescent leaf (LS) were used (Supplementary Figure S2) and all of the $15 N t N A C$ genes tested were found to be up-regulated during senescence, with a fold change of two or more (Figure 4). Among them, NtNACs including NtNAC002, NtNAC030, NtNAC046, NtNAC079, NtNAC098, and NtNAC149 were up-regulated at the MS stage with high level expression maintained until the LS stage. Expression of some other NtNACs genes (NtNAC008, $N t N A C 073, N t N A C 099$, and NtNAC148) were induced only at the LS stage. Expression of NtNAC028, NtNAC080, NtNAC083, and $N t N A C 117$ increased rapidly at the ES stage, suggesting potential roles of these genes in regulating the onset of leaf senescence. Phylogenetic analysis showed that NtNAC028, NtNAC080, and NtNAC083 were closely related to ANAC029/AtNAP, which acts as a key positive regulator of leaf senescence (Guo and Gan, 2006). Similarly, NtNAC117 was in the same clade that contained the Arabidopsis senescence-associated genes ANAC059/ORS1 and ANAC092/ORE1, which also play significant roles in regulating senescence (Balazadeh et al., 2010, 2011; Rauf et al., 2013). We therefore predicted that these four genes (NtNAC028, NtNAC080, $N t N A C 083$, and $N t N A C 117)$ might function as key regulators of leaf senescence in tobacco.

\section{Overexpression of NtNAC 080 Induces Early Leaf Senescence in Arabidopsis}

To further assess the function of senescence-associated NtNAC genes in planta, we obtained transgenic Arabidopsis plants overexpressing NtNAC080, expression of which increased significantly at early stages of leaf senescence in tobacco, with a 48-folds change at the ES stage. Two lines of NtNAC080overexpressing transgenic Arabidopsis, with expression of NtNAC080 confirmed by qRT-PCR (Figure 5D), exhibited a premature senescence at 45 days after sowing (DAS) (Figure 5A). The precocious leaf yellowing phenotype was also supported by changes in total chlorophyll content and the maximal photochemical efficiency of PSII (Fv/Fm), which reflects the photochemical quantum efficiency of PSII and photosynthetic activity (Figures 5B,C). Expression of the senescence-specific cysteine protease $S A G 12$, which is a widely used molecular marker of leaf senescence, was strongly induced in leaves of the two NtNAC080-overexpressing lines (Figure 5E). These data indicated that, like its close homolog ANAC029/AtNAP in the NAC-f subgroup, NtNAC080 also acts as a positive regulator of leaf senescence.

\section{CRISPR-Mediated NtNAC080 Mutations Delay Leaf Senescence in Tobacco}

To confirm the function of NtNACO80 in regulating tobacco leaf senescence, we mutated NtNAC080 using the CRISPR/Cas9 system in tobacco. Two gRNAs (sgRNA1 and sgRNA2) targeting the NAC domain of NtNAC080 were created and used in Agrobacterium-mediated transformation of tobacco plants (Figure 6A). Twelve T0 plants for NtNAC080 sgRNA1 and 14 T0 plants for NtNAC080 sgRNA2 were obtained after kanamycin screening. PCR-amplified target regions of these plants were sequenced to identify mutations. Eleven of the $12(91.7 \%)$ sgRNA1 and 9 of the 14 (64.3\%) sgRNA2 T0 plants were found to contain mutations with deletion or insertion of nucleotides (Supplementary Table S7). Homozygous mutant plants were 


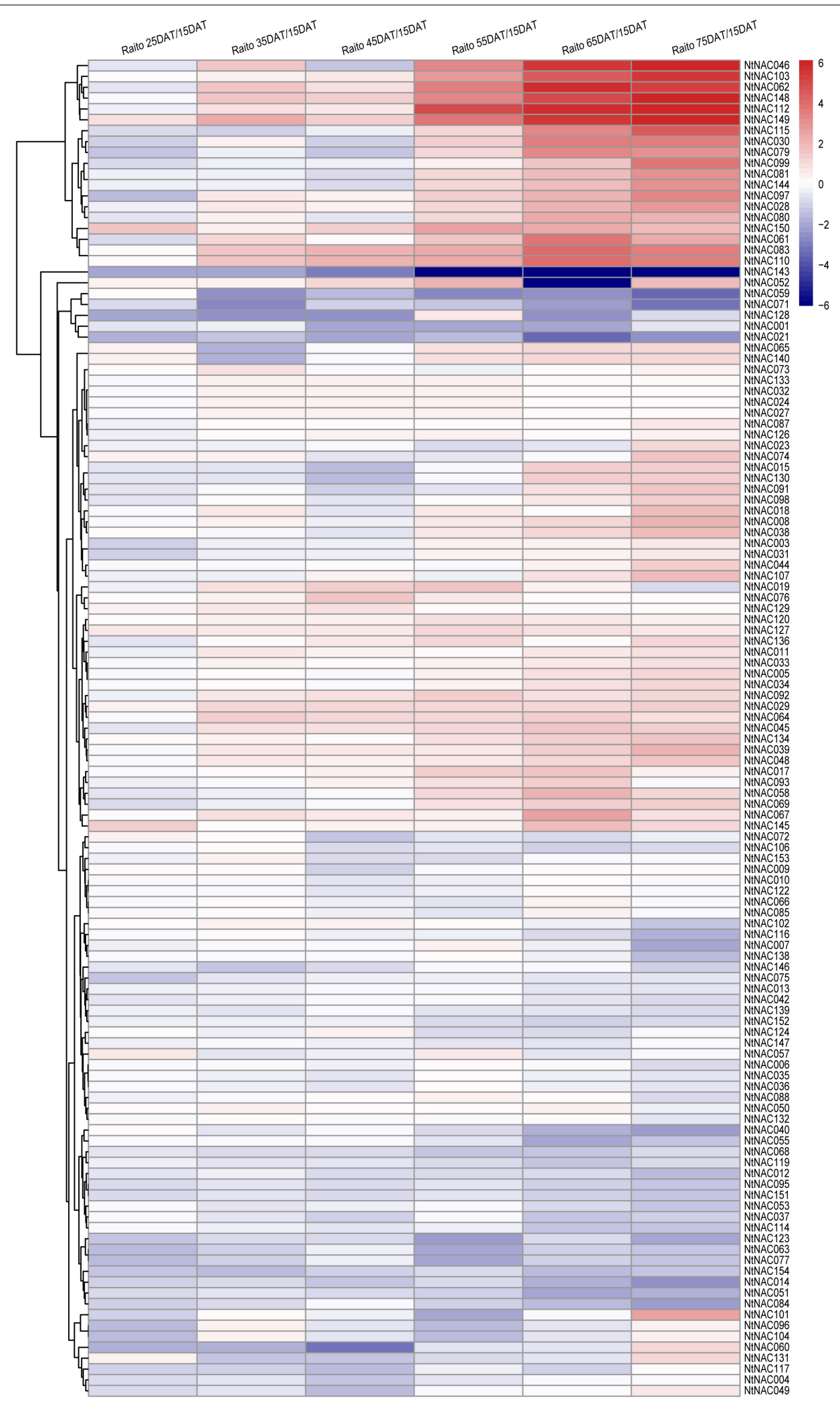

FIGURE 3 | Heatmap representation and hierarchical clustering of NtNAC genes at different stages of leaf senescence. The lllumina RNA-Seq data was reanalyzed, and the heatmap was generated based on the log2 fold-change values at 25/35/45/55/65/75DAT when compared with 15DAT. Changes in expression levels were displayed from blue (down-regulated) to red (up-regulated), as shown in the color gradient at the top right corner. DAT, days after topping. 

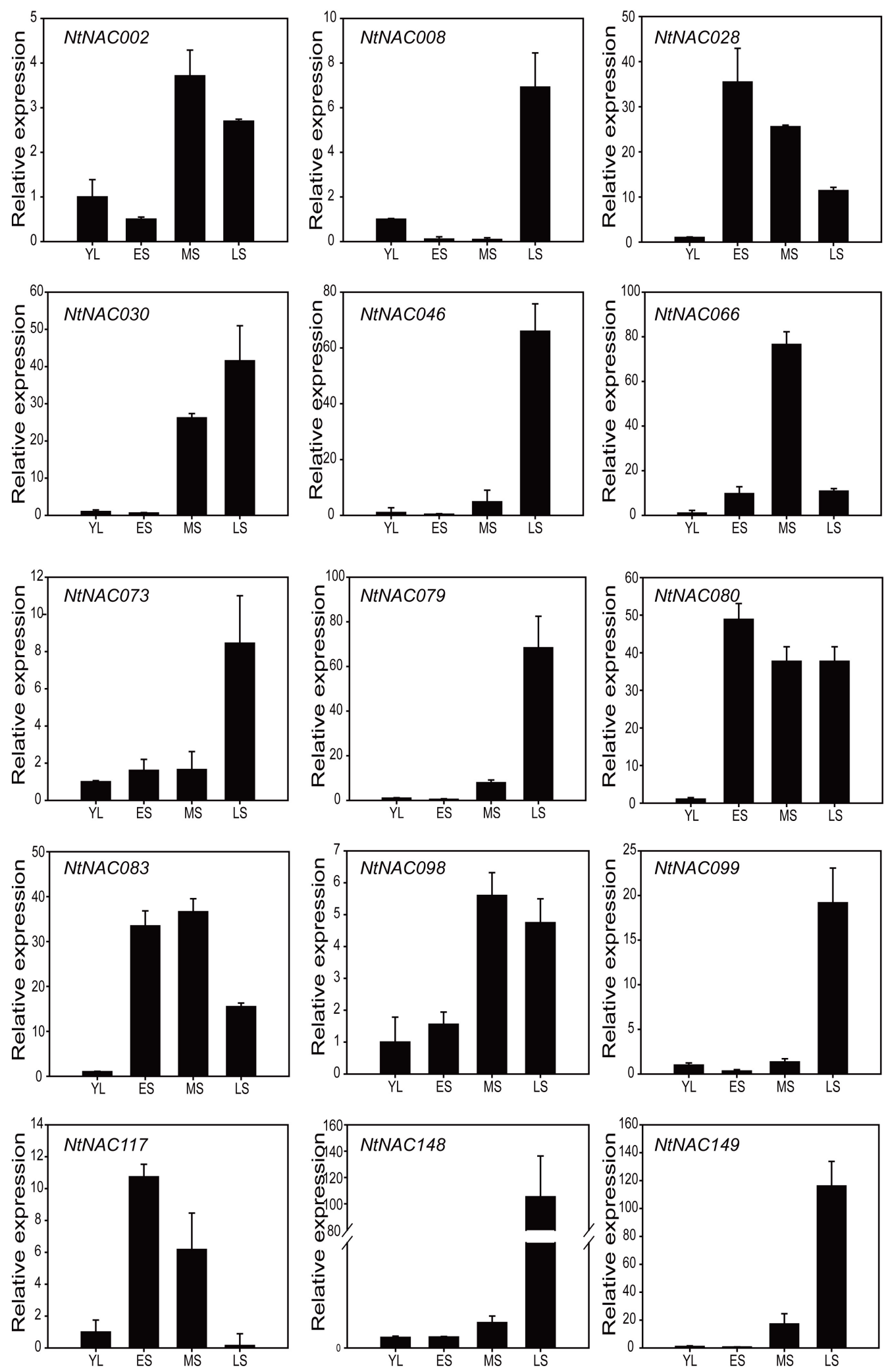

FIGURE 4 | Expression profiles of 15 representative NtNAC genes analyzed by qRT-PCR during tobacco leaf senescence. The relative expression ratio of each gene was calculated relative to its expression at the first stage (YL). qRT-PCR data were normalized using tobacco Actin gene. The bars are standard deviations (SD) of three biological replicates. 
A

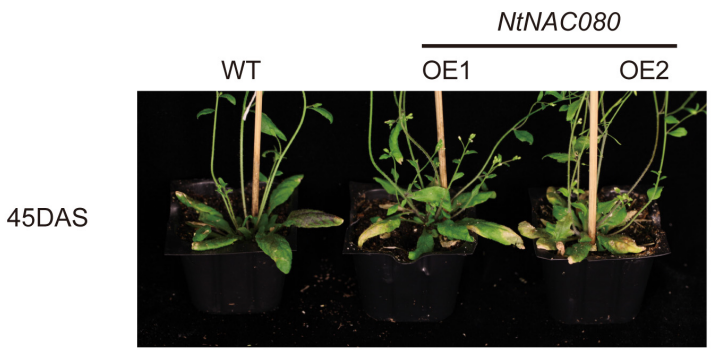

B

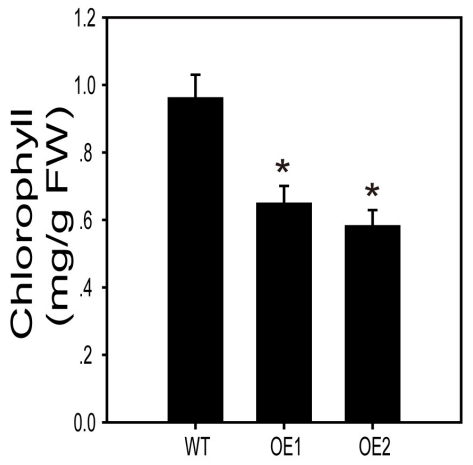

D

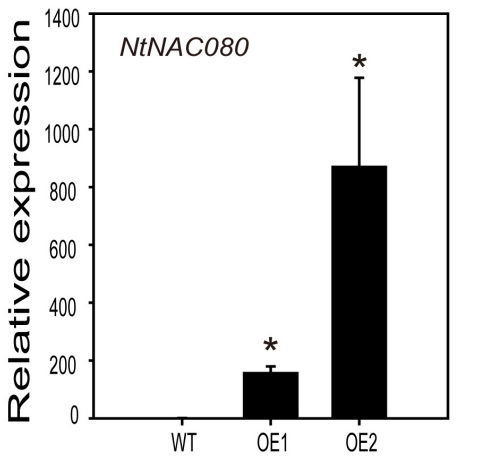

C

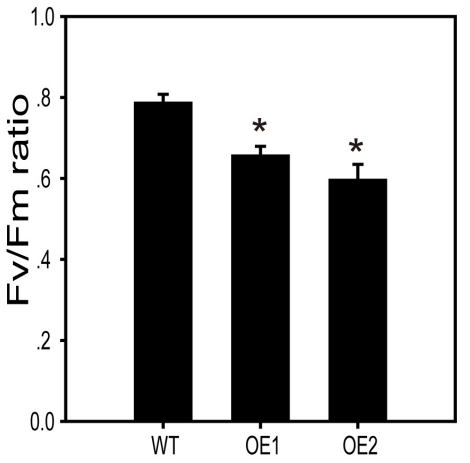

$E$

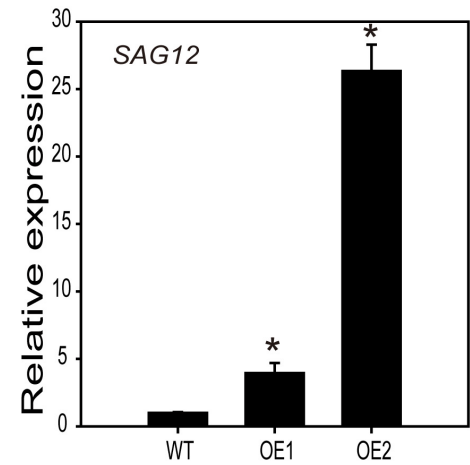

FIGURE 5 | Overexpression of NtNACO80 in Arabidopsis plants causes precocious senescence. (A) Phenotypes of wild-type (WT) and transgenic plants (OE1 and OE2). The picture was taken at 45 days after sowing. (B,C) Chlorophyll content and Fv/Fm ratios in leaf 5 of age-matched WT and two OE lines (leaf number counted from the bottom of the plant). (D,E) qRT-PCR analysis of expression of NtNACO80 and SAG12 in WT and two OE lines. AtActin was used as the internal control. The bars are standard deviations (SD) of three biological replicates. *Significant difference using student's $t$-test $(p<0.05)$.

obtained from the $\mathrm{T} 1$ generation and two representative lines ntnac080-1 and ntnac080-2, were further used for phenotype analysis (Figure 6C). The ntnac080-1 line had a 1 bp insertion at the $3^{\prime}$ end of sgRNA1 sequence which introduced a stop codon after position $\mathrm{P} 121$, and the ntnac080-2 plants harbor a $12 \mathrm{bp}$ deletion causing a deletion of four amino acids and transition of one amino acid at position 117 (Figure 6B). In addition, we have also sequenced NtNAC028, the closest homolog of NtNAC080, in the two ntnac080 mutants. The sequencing results confirmed that these CRISPR mutants contain no mutation on NtNAC028 gene. Thus these mutant lines might express abnormal NtNAC080 proteins with a truncated/mutated NAC domain (Figures 6B,C). Both ntnac080-1 and ntnac080-2 plants showed a similar delayed senescence phenotype compared to WT (K326) tobacco (Figure 6D). Quantitative RT-PCR indicated no difference in transcript levels of NtNAC080 between these two mutants and WT plants (Figure 6E). We also assessed the progression of leaf senescence between mutants and WT by measuring changes in chlorophyll content and the maximal photochemical efficiency of PSII (Fv/Fm). Under glasshouse conditions, chlorophyll levels in individual leaves (leaf 1-6, numbered from the top to the bottom of a plant) of the two mutants were generally higher than that in counterpart leaves of the age-matched WT plants (Figure 6F). The Fv/Fm ratios in leaf 5 and 6 of the mutants plants were also higher than that in counterpart leaves of WT plants (Figure 6G). Furthermore, we determined the expression of senescence-related marker genes NtCP1 (SAG12 homolog in tobacco) and NtRBCS in leaf 5. As shown in Figures $\mathbf{6 H}, \mathbf{I}$, the expression of $N t C P 1$ was lower and $N t R B C S$ was higher in two mutants. These data indicated that NtNAC080 acts as a positive regulator of leaf senescence in tobacco. 


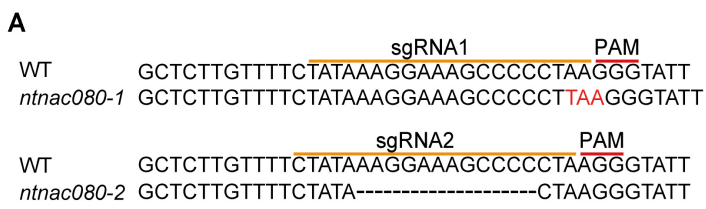

B
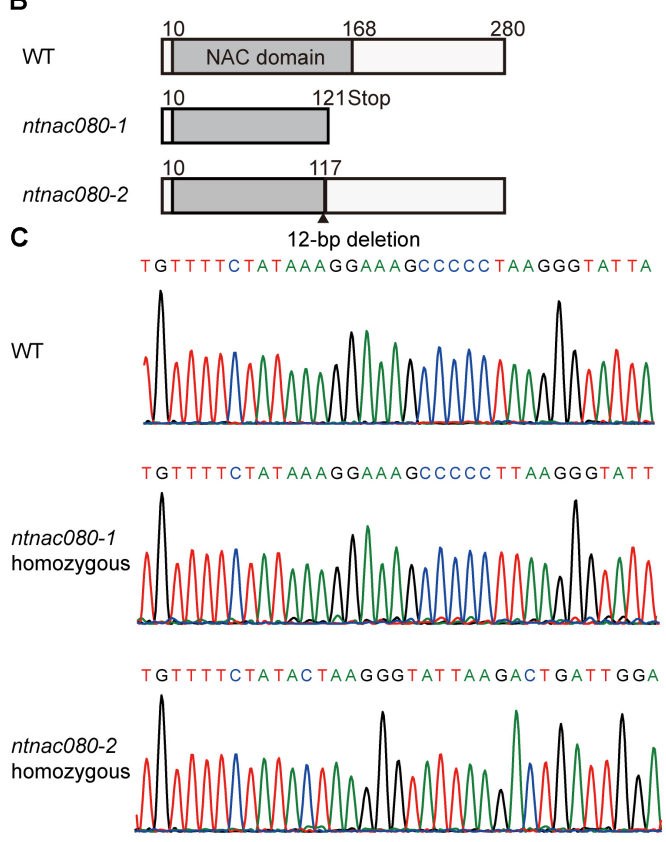

D

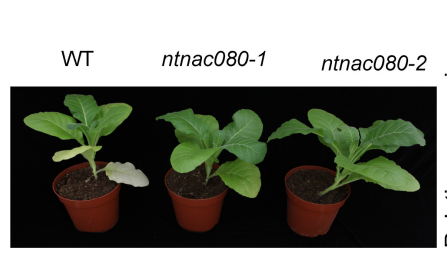

F

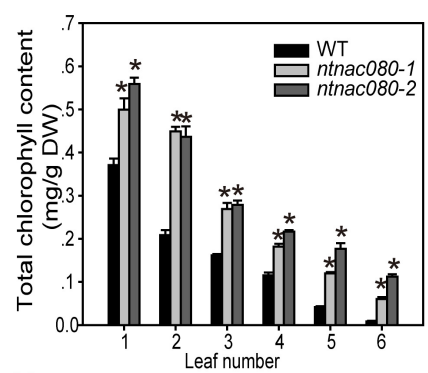

H

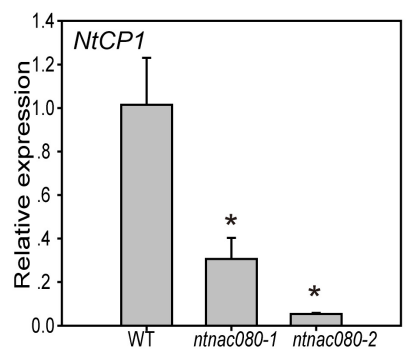

E
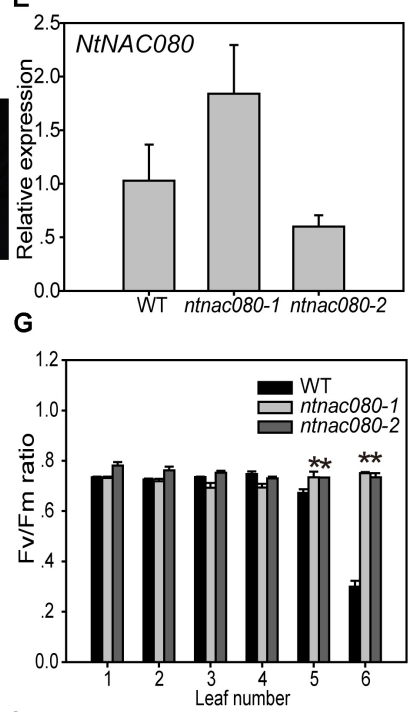

I

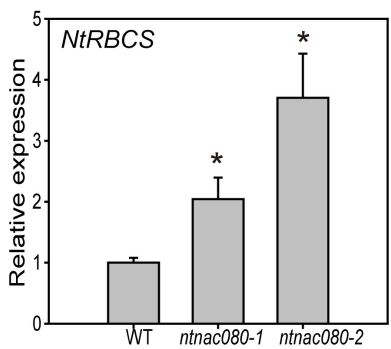

FIGURE 6 | Delayed leaf senescence phenotype of the CRISPR-mediated ntnac080 mutant compared with that of age-matched wild-type plants. (A-C) The construction of NtNAC080 CRISPR-Cas9 lines. In (A,C) sequence results of mutants were aligned to the reference genome sequence. In (B) putative mutated NtNAC080 proteins in homozygous plants (T1). Gray zones were NAC conserved domains (10-168 aa). In ntnac080-1 plants, 1 bp insertion led to a stop after position P121. In ntnac080-2 plants, a 12 bp deletion caused a deletion of four amino acids and transition of one amino acid at position 117. (D) Phenotypes of ntnac080 mutants. (E) qRT-PCR analysis of expression of NtNAC080 in ntnac080-1 mutants. (F,G) Chlorophyll content and Fv/Fm ratios in leaf 1-6 of age-matched wild-type (K326) and mutant lines (leaf number counted from the top of plant). (H,I) qRT-PCR analysis of expression of NtCP1 and NtRBCS genes in leaf 5 of wild-type (K326) and mutant lines. NtActin was used as the internal control. The bars are standard deviations (SD) of three biological replicates. $*$ Significant difference using student's $t$-test $(p<0.05)$.

\section{DISCUSSION}

As one of the most important transcription factor families in plant, the NAC gene family plays a pivotal role in regulating various development and physiological processes. Although the functions of a number of NAC genes have been investigated in Arabidopsis and model crops (Guo and Gan, 2006; Garapati et al., 2015; Pimenta et al., 2016; Mao et al., 2017), little is known about this gene family in the economic crop Nicotiana tabacum. An earlier study has identified 152 NAC domain genes from methylation filtrated tobacco genomic DNA libraries but detailed information of this gene family was not available (Rushton et al., 2008). The biological functions of most tobacco NAC genes remain unknown except two NAC genes that have been functionally characterized (Fu et al., 2013; Han et al., 2014). In this study we identified 154 NAC proteins in the tobacco genome and compared them with the Arabidopsis NAC proteins. In many cases two or more tobacco NAC proteins were found to be highly homologous to an Arabidopsis NAC, most likely due to the whole-genome duplication during tobacco tetraploidization. The duplicated genes may have evolved to have diversified functions.

Based on phylogenic analysis, the NAC proteins from tobacco and Arabidopsis were divided into 15 distinct subgroups. The exon/intron structure and motif compositions of NACs were highly conserved in each subgroup. Earlier studies suggested that NAC genes from the same subgroup tend to have similar functions (Fang et al., 2008; Hu et al., 2015; Wei et al., 2016). For instance, subgroup NAC-i contains most of membranebound NAC members of tobacco and Arabidopsis, suggesting a potential role of the subgroup NAC-i members in regulating stress responses.

Two other subgroups of the NAC family proteins, namely subgroup NAC-b and $\mathrm{f}$, seem to have an important role in regulating leaf senescence. A great numbers of the senescence up-regulated ANACs are clustered in subgroups NAC-b and NAC-f, and a number of previously characterized senescenceregulating ANAC TFs are also in these subgroups (Figure 1). We examined the expression of $15 N t N A C$ genes in subgroups 
NAC-b and NAC-f via qRT-PCR analysis and all of these NtNAC genes showed a significant up-regulation during tobacco leaf senescence (Figure 4).

The role of NAC family TFs in leaf senescence seems to be conserved between different plant species. In Arabidopsis, 30 out of 105 NAC genes are up-regulated during leaf senescence, including AtNAP, ORE1, and ORS1 (Breeze et al., 2011). In barley, $\sim 15$ out of 47 studied NAC genes are up-regulated during senescence (Christiansen and Gregersen, 2014). Phylogenetic analysis of the barley NAC proteins suggested that 12 of these 15 up-regulated NAC genes belonged to two subfamilies, which also harbor a number of previously characterized senescenceregulating NAC TFs from other species, such as Arabidopsis, rice, and wheat. Based on sequence homology and expression profiles, several putative regulators of senescence in barley, including HvNAC005, HvNAC027, and HvNAC029/HvNAM1, have been identified (Christiansen et al., 2011; Podzimska-Sroka et al., 2015). Interestingly, majority of the ANAC genes in these two above-mentioned barley NAC subfamilies also belong to NAC-b and $\mathrm{f}$ in the phylogenetic tree in this study (Figure 1).

A number of studies have shown that NAC genes are induced in senescent leaves and that overexpression of some $N A C$ genes alters senescence process in plants. In Arabidopsis, overexpression of either AtNAP or ORE1 promoted leaf senescence, while overexpression of JUB1 or VNI2 led to delayed senescence (Guo and Gan, 2006; Yang et al., 2011; Wu et al., 2012; Rauf et al., 2013). In rice, overexpression of OsNAC2 and OsNAP resulted in early senescence, while overexpression of OsNAC106 resulted in delayed senescence (Liang et al., 2014; Sakuraba et al., 2015; Mao et al., 2017). Meanwhile, a wheat NAC transcription factor, $N A M-B 1$, accelerates senescence and increases nutrient remobilization from leaves to developing grain in wheat (Uauy et al., 2006). It is noteworthy that the genes which promote the process of leaf senescence, such as AtNAP, OsNAP, NAM$B 1, O R E 1$, and OsNAC2, were phylogenetically clustered together (Podzimska-Sroka et al., 2015), indicating that the NAC genes with similar biological functions are closely related.

However, the genes that function in delaying senescence were found randomly distributed on the phylogenetic tree. For example, VNI2 was a member of the subgroup NAC-e while JUB1 belonged to the NAC-h subgroup (Figure 1). Many of the NAC genes in the NAC-e subgroup were up-regulated while genes of the NAC-h subgroup were down-regulated in senescent leaves (Supplementary Tables S5, S6). Four NtNACs genes, including NtNAC004, NtNAC049, NtNAC015, and NtNAC130 were closely related to the negative senescence regulator VNI2. Similarly, NtNAC146 was clustered in the same clade with JUB1. At the transcriptional level, NtNAC049, NtNAC015, and NtNAC130 were down-regulated at the early stage of senescence (from 15DAT to 55DAT) then up-regulated after 65DAT. Whereas, NtNAC004 and NtNAC146 were continuously down-regulated during leaf senescence. In Arabidopsis, JUB1 and VNI2 showed significantly up-regulation during senescence. The difference in expression patterns of these NAC genes between tobacco and Arabidopsis suggests that in comparison with their Arabidopsis homologs, the negative senescence regulators in the tobacco NAC family might have different mechanisms in controlling leaf senescence. The senescence-inhibiting NAC genes may have more complex evolutionary relationships compared to the senescence-promoting NACs.

In the current study, to explore the function of NtNACs in regulating leaf senescence, we have identified orthologous pairs between NtNACs and ANACs based on sequence similarity. Due to the polyploid genome of common tobacco, we have generally identified two or more NtNACs highly homologous to each Arabidopsis NAC gene. For instance, two homologous gene pairs (NtNAC028/NtNAC080 and NtNAC083/NtNAC110) were found to be orthologous to the key senescence- regulating AtNAP. The tobacco NAP homologs exhibited similar patterns of transcript accumulation during leaf senescence (Figure 3). Furthermore, NtNAC028, NtNAC080, and NtNAC083 were induced rapidly at early senescence (ES) stage in the qRTPCR analysis, indicating that these genes may perform similar function as AtNAP in regulating the onset of leaf senescence. To validate this hypothesis, we carried out functional analysis of some of these genes. Our results showed that overexpression of NtNAC080 induced Arabidopsis early leaf senescence, whereas ntnac080 mutants obtained via CRISPR-Cas9 strategy delayed leaf senescence of tobacco leaves. Similar results were obtained for NtNAC028 overexpressing and knocking-out studies in our laboratory (unpublished data). The deduced proteins of NtNAC028 and NtNAC080 are closely related to each other (91.87\% sequence identity). Overexpression of both NtNAC028 and NtNAC080 induced senescence while mutation in either gene caused delay in leaf senescence (Figures 5, 6, unpublished data), suggesting that as a result of gene family expansion in tobacco, these homologous NtNAP genes share similar but not completely redundant roles in regulating leaf senescence. This may lead to more plasticity in senescence regulation. Like NtNAC028/NtNAC080, expression the other homologous pair (NtNAC083/ NtNAC110) was also found increased during leaf senescence, but the transcripts of these two genes showed an obvious increase at 35DAT, before the initiation of leaf senescence (Figure 3). The difference in expression patterns suggested that NtNAC083 and NtNAC110 may play distinct roles in regulating leaf senescence and other development processes in tobacco. Further investigation is needed in elucidating the molecular mechanisms underlying the regulation of leaf senescence by NtNAP genes as well as other NAC family genes in tobacco.

Overall, this study demonstrates that a number of NAC genes might be involved in regulating leaf senescence in tobacco and have revealed a potential structure-function relationship between NAC family members as putative senescence regulators.

\section{AUTHOR CONTRIBUTIONS}

YG conceived the project. YG and WL designed the research. WL performed plant transformation, the phenotypic analysis, and qRT-PCR verification. XL performed NtNAC TF identification, phylogenetic analysis, gene structure, and motif analysis. WL, XL, and JC performed transcriptomic analyses. ZZ and WW designed CRISPR-Cas9 experiment and generated the constructs. WL, YG, and XL wrote the manuscript. 


\section{FUNDING}

This work was financially supported by the Fundamental Research Funds for Chinese Academy of Agricultural Sciences (2013ZL024) and the Agricultural Science and Technology Innovation Program (ASTIP-TRIC02).

\section{SUPPLEMENTARY MATERIAL}

The Supplementary Material for this article can be found online at: https://www.frontiersin.org/articles/10.3389/fpls.2018.01900/ full\#supplementary-material

FIGURE S1 | Sequence logos for conserved motifs identified in NtNACs by MEME analysis.

\section{REFERENCES}

Ali, A., Gao, X., and Guo, Y. (2018). Initiation, progression, and genetic manipulation of leaf senescence. Methods Mol. Biol. 1744, 9-31. doi: 10.1007/ 978-1-4939-7672-0_2

Apweiler, R., Attwood, T. K., Bairoch, A., Bateman, A., Birney, E., Biswas, M., et al. (2001). The InterPro database, an integrated documentation resource for protein families, domains and functional sites. Nucleic Acids Res. 29, 37-40.

Bailey, T. L., Johnson, J., Grant, C. E., and Noble, W. S. (2015). The MEME suite. Nucleic Acids Res. 43, W39-W49. doi: 10.1093/nar/gkv416

Balazadeh, S., Kwasniewski, M., Caldana, C., Mehrnia, M., Zanor, M. I., Xue, G.-P., et al. (2011). ORS1, an $\mathrm{H}(2) \mathrm{O}(2)$-responsive NAC transcription factor, controls senescence in Arabidopsis thaliana. Mol. Plant 4, 346-360. doi: 10.1093/mp/ ssq080

Balazadeh, S., Riaño-Pachón, D. M., and Mueller-Roeber, B. (2008). Transcription factors regulating leaf senescence in Arabidopsis thaliana. Plant Biol. 10, 63-75. doi: 10.1111/j.1438-8677.2008.00088.x

Balazadeh, S., Siddiqui, H., Allu, A. D., Matallana-Ramirez, L. P., Caldana, C., Mehrnia, M., et al. (2010). A gene regulatory network controlled by the NAC transcription factor ANAC092/AtNAC2/ORE1 during salt-promoted senescence. Plant J. 62, 250-264. doi: 10.1111/j.1365-313X.2010.04151.x

Besseau, S., Li, J., and Palva, E. T. (2012). WRKY54 and WRKY70 co-operate as negative regulators of leaf senescence in Arabidopsis thaliana. J. Exp. Bot. 63, 2667-2679. doi: 10.1093/jxb/err450

Breeze, E., Harrison, E., McHattie, S., Hughes, L., Hickman, R., Hill, C., et al. (2011). High-resolution temporal profiling of transcripts during Arabidopsis leaf senescence reveals a distinct chronology of processes and regulation. Plant Cell 23, 873-894. doi: 10.1105/tpc.111.083345

Chai, M., Bellizzi, M., Wan, C., Cui, Z., Li, Y., and Wang, G.-L. (2015). The NAC transcription factor OsSWN1 regulates secondary cell wall development in Oryza sativa. J. Plant Biol. 58, 44-51. doi: 10.1007/s12374-014-0400-y

Chen, Y., Qiu, K., Kuai, B., and Ding, Y. (2011). Identification of an NAP-like transcription factor BeNAC1 regulating leaf senescence in bamboo (Bambusa emeiensis 'Viridiflavus'). Physiol. Plant. 142, 361-371. doi: 10.1111/j.1399-3054. 2011.01472.x

Christiansen, M. W., and Gregersen, P. L. (2014). Members of the barley NAC transcription factor gene family show differential co-regulation with senescence-associated genes during senescence of flag leaves. J. Exp. Bot. 65, 4009-4022. doi: 10.1093/jxb/eru046

Christiansen, M. W., Holm, P. B., and Gregersen, P. L. (2011). Characterization of barley (Hordeum vulgare L.) NAC transcription factors suggests conserved functions compared to both monocots and dicots. BMC Res. Notes 4:302. doi: 10.1186/1756-0500-4-302

Clough, S. J., and Bent, A. F. (1998). Floral dip: a simplified method for Agrobacterium-mediated transformation of Arabidopsis thaliana. Plant J. 16, 735-743. doi: 10.1046/j.1365-313x.1998.00343.x
FIGURE S2 | Senescence stages of tobacco leaves used in this study. YL, young leaf; ES, an early senescent leaf; MS, a mid-senescent leaf; LS, a late senescent leaf.

TABLE S1 | Sequences of primer pairs used for qRT-PCR analysis.

TABLE S2 | Sequences of primer pairs used for vector construction and transgenic plants sequencing.

TABLE S3 | Characteristics of NAC gene family in tobacco.

TABLE S4 | Protein sequences of NAC gene family in tobacco.

TABLE S5 | Expression change ratio of ANAC genes between senescent leaves and non-senescing leaves.

TABLE S6 | Expression Patterns of 154 NtNACs during tobacco leaf senescence.

TABLE S7 | Summary of targeted mutagenesis at the NtNAC080 locus in transgenic plants (TO).

Ernst, H. A., Nina Olsen, A., Skriver, K., Larsen, S., and Lo Leggio, L. (2004). Structure of the conserved domain of ANAC, a member of the NAC family of transcription factors. EMBO Rep. 5, 297-303. doi: 10.1038/sj.embor.7400093

Fan, K., Bibi, N., Gan, S., Li, F., Yuan, S., Ni, M., et al. (2015). A novel NAP member GhNAP is involved in leaf senescence in Gossypium hirsutum. J. Exp. Bot. 66, 4669-4682. doi: 10.1093/jxb/erv240

Fang, Y., You, J., Xie, K., Xie, W., and Xiong, L. (2008). Systematic sequence analysis and identification of tissue-specific or stress-responsive genes of NAC transcription factor family in rice. Mol. Genet. Genomics 280, 547-563. doi: 10.1007/s00438-008-0386-6

Fu, Y., Guo, H., Cheng, Z., Wang, R., Li, G., Huo, G., et al. (2013). NtNAC-R1, a novel NAC transcription factor gene in tobacco roots, responds to mechanical damage of shoot meristem. Plant Physiol. Biochem. 69, 74-81. doi: 10.1016/j. plaphy.2013.05.004

Gao, J., Wang, G., Ma, S., Xie, X., Wu, X., Zhang, X., et al. (2015). CRISPR/Cas9mediated targeted mutagenesis in Nicotiana tabacum. Plant Mol. Biol. 87, 99-110. doi: 10.1007/s11103-014-0263-0

Garapati, P., Xue, G.-P., Munné-Bosch, S., and Balazadeh, S. (2015). Transcription factor ATAF1 in Arabidopsis promotes senescence by direct regulation of key chloroplast maintenance and senescence transcriptional cascades. Plant Physiol. 168, 1122-1139. doi: 10.1104/pp.15.00567

Gerstel, D. U. (1960). Segregation in new allopolyploids of Nicotiana. I. Comparison of $6 \times(\mathrm{N}$. tabacum $\times$ tomentosiformis $)$ and $6 \times(\mathrm{N}$. tabacum $\times$ otophora). Genetics 45, 1723-1734.

Gregersen, P. L., and Holm, P. B. (2007). Transcriptome analysis of senescence in the flag leaf of wheat (Triticum aestivum L.). Plant Biotechnol. J. 5, 192-206. doi: 10.1111/j.1467-7652.2006.00232.x

Guo, Y. (2013). Towards systems biological understanding of leaf senescence. Plant Mol. Biol. 82, 519-528. doi: 10.1007/s11103-012-9974-2

Guo, Y., Cai, Z., and Gan, S. (2004). Transcriptome of Arabidopsis leaf senescence. Plant Cell Environ. 27, 521-549. doi: 10.1111/j.1365-3040.2003.01158.x

Guo, Y., and Gan, S. (2006). AtNAP, a NAC family transcription factor, has an important role in leaf senescence. Plant J. 46, 601-612. doi: 10.1111/j.1365313X.2006.02723.x

Guo, Y., and Gan, S. (2012). Convergence and divergence in gene expression profiles induced by leaf senescence and 27 senescence-promoting hormonal, pathological and environmental stress treatments. Plant Cell Environ. 35, 644655. doi: 10.1111/j.1365-3040.2011.02442.x

Guo, Y., and Gan, S. S. (2014). Translational researches on leaf senescence for enhancing plant productivity and quality. J. Exp. Bot. 65, 3901-3913. doi: 10. 1093/jxb/eru248

Hajdukiewicz, P., Svab, Z., and Maliga, P. (1994). The small, versatile pPZP family of Agrobacterium binary vectors for plant transformation. Plant Mol. Biol. 25, 989-994. doi: 10.1007/BF00014672

Hajouj, T., Michelis, R., and Gepstein, S. (2000). Cloning and characterization of a receptor-like protein kinase gene associated with senescence. Plant Physiol. 124, 1305-1314. doi: 10.1104/pp.124.3.1305 
Han, Q. Q., Qiao, P., Song, Y. Z., and Zhang, J. Y. (2014). Structural analysis and tissue-specific expression patterns of a novel salt-inducible NAC transcription factor gene from Nicotiana tabacum cv. Xanthi. J. Hortic. Sci. Biotechnol. 89, 700-706. doi: 10.1080/14620316.2014.11513140

He, Y., and Gan, S. (2002). A gene encoding an acyl hydrolase is involved in leaf senescence in Arabidopsis. Plant Cell 14, 805-815. doi: 10.1105/tpc.010422

Horsch, R. B., Fry, J., Hoffmann, N., Neidermeyer, J., Rogers, S. G., and Fraley, R. T. (1989). "Leaf disc transformation," in Plant Molecular Biology Manual, eds S. B. Gelvin, R. A. Schilperoort, and D. P. S. Verma (Dordrecht: Springer), 63-71.

Hu, R., Qi, G., Kong, Y., Kong, D., Gao, Q., and Zhou, G. (2010). Comprehensive analysis of NAC domain transcription factor gene family in Populus trichocarpa. BMC Plant Biol. 10:145. doi: 10.1186/1471-2229-10-145

Hu, W., Wei, Y., Xia, Z., Yan, Y., Hou, X., Zou, M., et al. (2015). Genome-wide identification and expression analysis of the NAC transcription factor family in cassava. PLoS One 10:e0136993. doi: 10.1371/journal.pone.0136993

Huang, Q., Wang, Y., Li, B., Chang, J., Chen, M., Li, K., et al. (2015). TaNAC29, a NAC transcription factor from wheat, enhances salt and drought tolerance in transgenic Arabidopsis. BMC Plant Biol. 15:268. doi: 10.1186/s12870-0150644-9

Jensen, M. K., Hagedorn, P. H., De Torres-Zabala, M., Grant, M. R., Rung, J. H., Collinge, D. B., et al. (2008). Transcriptional regulation by an NAC (NAMATAF1,2-CUC2) transcription factor attenuates ABA signalling for efficient basal defence towards Blumeria graminis f. sp. hordei in Arabidopsis. Plant J. 56, 867-880. doi: 10.1111/j.1365-313X.2008.03646.x

Johnson, L. S., Eddy, S. R., and Portugaly, E. (2010). Hidden markov model speed heuristic and iterative HMM search procedure. BMC Bioinformatics 11:431. doi: 10.1186/1471-2105-11-431

Katoh, K., and Standley, D. M. (2013). MAFFT multiple sequence alignment software version 7: improvements in performance and usability. Mol. Biol. Evol. 30, 772-780. doi: 10.1093/molbev/mst010

Kim, S.-G., Lee, S., Seo, P. J., Kim, S.-K., Kim, J.-K., and Park, C.-M. (2010). Genome-scale screening and molecular characterization of membrane-bound transcription factors in Arabidopsis and rice. Genomics 95, 56-65. doi: 10.1016/ j.ygeno.2009.09.003

Kim, S.-Y., Kim, S.-G., Kim, Y.-S., Seo, P. J., Bae, M., Yoon, H.-K., et al. (2007). Exploring membrane-associated NAC transcription factors in Arabidopsis: implications for membrane biology in genome regulation. Nucleic Acids Res. 35, 203-213. doi: 10.1093/nar/gkl1068

Kim, Y.-S., Kim, S.-G., Park, J.-E., Park, H.-Y., Lim, M.-H., Chua, N.-H., et al. (2006). A membrane-bound NAC transcription factor regulates cell division in Arabidopsis. Plant Cell 18, 3132-3144. doi: 10.1105/tpc.106.043018

Kjaersgaard, T., Jensen, M. K., Christiansen, M. W., Gregersen, P., Kragelund, B. B., and Skriver, K. (2011). Senescence-associated barley NAC (NAM, ATAF1,2, CUC) transcription factor interacts with radical-induced cell death 1 through a disordered regulatory domain. J. Biol. Chem. 286, 35418-35429. doi: 10.1074/ jbc.M111.247221

Krogh, A., Larsson, B., von Heijne, G., and Sonnhammer, E. L. L. (2001). Predicting transmembrane protein topology with a hidden markov model: application to complete genomes. J. Mol. Biol. 305, 567-580. doi: 10.1006/jmbi.2000.4315

Le, D. T., Nishiyama, R., Watanabe, Y., Mochida, K., Yamaguchi-Shinozaki, K., Shinozaki, K., et al. (2011). Genome-wide survey and expression analysis of the plant-specific NAC transcription factor family in soybean during development and dehydration stress. DNA Res. 18, 263-276. doi: 10.1093/dnares/dsr015

Lee, S., Seo, P. J., Lee, H.-J., and Park, C.-M. (2012). A NAC transcription factor NTL4 promotes reactive oxygen species production during drought-induced leaf senescence in Arabidopsis. Plant J. 70, 831-844. doi: 10.1111/j.1365-313X. 2012.04932.x

Li, Q., Lin, Y.-C., Sun, Y.-H., Song, J., Chen, H., Zhang, X.-H., et al. (2012). Splice variant of the SND1 transcription factor is a dominant negative of SND1 members and their regulation in Populus trichocarpa. Proc. Natl. Acad. Sci. U.S.A. 109, 14699-14704. doi: 10.1073/pnas.1212977109

Li, S., Wang, N., Ji, D., Xue, Z., Yu, Y., Jiang, Y., et al. (2016). Evolutionary and functional analysis of membrane-bound NAC transcription factor genes in soybean. Plant Physiol. 172, 1804-1820. doi: 10.1104/pp.16.01132

Li, W., and Guo, Y. (2018). RNA-Seq analysis of the transcriptome of leaf senescence in tobacco. Methods Mol. Biol. 1744, 331-337. doi: 10.1007/978-14939-7672-0_27
Li, W., and Guo, Y. F. (2014). Transcriptome, transcription factors and transcriptional regulation of leaf senescence. J. Bioinformatics Comp. Genomics $1,1-3$.

Li, W., Zhang, H., Li, X., Zhang, F., Liu, C., Du, Y., et al. (2017). Intergrative metabolomic and transcriptomic analyses unveil nutrient remobilization events in leaf senescence of tobacco. Sci. Rep. 7:12126. doi: 10.1038/s41598-01711615-0

Liang, C., Wang, Y., Zhu, Y., Tang, J., Hu, B., Liu, L., et al. (2014). OsNAP connects abscisic acid and leaf senescence by fine-tuning abscisic acid biosynthesis and directly targeting senescence-associated genes in rice. Proc. Natl. Acad. Sci. U.S.A. 111, 10013-10018. doi: 10.1073/pnas.1321568111

Lim, P. O., Kim, H. J., and Nam, H. G. (2007). Leaf Senescence. Annu. Rev. Plant Biol. 58, 115-136. doi: 10.1146/annurev.arplant.57.032905.105316

Lindemose, S., Jensen, M. K., de Velde, J. V., O'Shea, C., Heyndrickx, K. S., Workman, C. T., et al. (2014). A DNA-binding-site landscape and regulatory network analysis for NAC transcription factors in Arabidopsis thaliana. Nucleic Acids Res. 42, 7681-7693. doi: 10.1093/nar/gku502

Ling, L., Song, L., Wang, Y., and Guo, C. (2017). Genome-wide analysis and expression patterns of the NAC transcription factor family in Medicago truncatula. Physiol. Mol. Biol. Plants 23, 343-356. doi: 10.1007/s12298-0170421-3

Lohman, K. N., Gan, S., John, M. C., and Amasino, R. M. (1994). Molecular analysis of natural leaf senescence in Arabidopsis thaliana. Physiol. Plant. 92, 322-328. doi: 10.1111/j.1399-3054.1994.tb05343.x

Mao, C., Lu, S., Lv, B., Zhang, B., Shen, J., He, J., et al. (2017). A Rice NAC transcription factor promotes leaf senescence via ABA biosynthesis. Plant Physiol. 174, 1747-1763. doi: 10.1104/pp.17.00542

Mitsuda, N., Iwase, A., Yamamoto, H., Yoshida, M., Seki, M., Shinozaki, K., et al. (2007). NAC transcription factors, NST1 and NST3, are key regulators of the formation of secondary walls in woody tissues of Arabidopsis. Plant Cell 19, 270-280. doi: 10.1105/tpc.106.047043

Mitsuda, N., and Ohme-Takagi, M. (2008). NAC transcription factors NST1 and NST3 regulate pod shattering in a partially redundant manner by promoting secondary wall formation after the establishment of tissue identity. Plant J. 56, 768-778. doi: 10.1111/j.1365-313X.2008.03633.x

Nakashima, K., Takasaki, H., Mizoi, J., Shinozaki, K., and Yamaguchi-Shinozaki, K. (2012). NAC transcription factors in plant abiotic stress responses. Biochim. Biophys. Acta 1819, 97-103. doi: 10.1016/j.bbagrm.2011.10.005

Nakashima, K., Tran, L.-S. P., Van Nguyen, D., Fujita, M., Maruyama, K., Todaka, D., et al. (2007). Functional analysis of a NAC-type transcription factor OsNAC6 involved in abiotic and biotic stress-responsive gene expression in rice. Plant J. 51, 617-630. doi: 10.1111/j.1365-313X.2007.03168.x

Nuruzzaman, M., Manimekalai, R., Sharoni, A. M., Satoh, K., Kondoh, H., Ooka, H., et al. (2010). Genome-wide analysis of NAC transcription factor family in rice. Gene 465, 30-44. doi: 10.1016/j.gene.2010.06.008

Olsen, A. N., Ernst, H. A., Leggio, L. L., and Skriver, K. (2005a). DNA-binding specificity and molecular functions of NAC transcription factors. Plant Sci. 169, 785-797. doi: 10.1016/j.plantsci.2005.05.035

Olsen, A. N., Ernst, H. A., Leggio, L. L., and Skriver, K. (2005b). NAC transcription factors: structurally distinct, functionally diverse. Trends Plant Sci. 10, 79-87. doi: 10.1016/j.tplants.2004.12.010

Ooka, H., Satoh, K., Doi, K., Nagata, T., Otomo, Y., Murakami, K., et al. (2003). Comprehensive analysis of NAC family genes in Oryza sativa and Arabidopsis thaliana. DNA Res. 10, 239-247. doi: 10.1093/dnares/10.6.239

Pimenta, M. R., Silva, P. A., Mendes, G. C., Alves, J. R., Caetano, H. D. N., Machado, J. P. B., et al. (2016). The stress-induced soybean NAC transcription factor GmNAC81 plays a positive role in developmentally programmed leaf senescence. Plant Cell Physiol. 57, 1098-1114. doi: 10.1093/pcp/pcw059

Podzimska-Sroka, D., O’Shea, C., Gregersen, P., and Skriver, K. (2015). NAC transcription factors in senescence: from molecular structure to function in crops. Plants 4, 412-448. doi: 10.3390/plants4030412

R Development Core Team (2013). R: A Language and Environment for Statistical Computing. Vienna: R Foundation for Statistical Computing, 12-21.

Rauf, M., Arif, M., Dortay, H., Matallana-Ramírez, L. P., Waters, M. T., Gil Nam, H., et al. (2013). ORE1 balances leaf senescence against maintenance by antagonizing G2-like-mediated transcription. EMBO Rep. 14, 382-388. doi: 10.1038/embor.2013.24 
Ríos, P., Argyris, J., Vegas, J., Leida, C., Kenigswald, M., Tzuri, G., et al. (2017). ETHQV6.3 is involved in melon climacteric fruit ripening and is encoded by a NAC domain transcription factor. Plant J. 91, 671-683. doi: 10.1111/tpj.13596

Rushton, P. J., Bokowiec, M. T., Han, S., Zhang, H., Brannock, J. F., Chen, X., et al. (2008). Tobacco transcription factors: novel insights into transcriptional regulation in the solanaceae. Plant Physiol. 147, 280-295. doi: 10.1104/pp.107. 114041

Sakuraba, Y., Piao, W., Lim, J.-H., Han, S.-H., Kim, Y.-S., An, G., et al. (2015). Rice ONAC106 inhibits leaf senescence and increases salt tolerance and tiller angle. Plant Cell Physiol. 56, 2325-2339. doi: 10.1093/pcp/pcv144

Sang-Gyu, K., An-Kyo, L., Hye-Kyung, Y., and Chung-Mo, P. (2008). A membrane-bound NAC transcription factor NTL8 regulates gibberellic acidmediated salt signaling in Arabidopsis seed germination. Plant J. 55, 77-88. doi: 10.1111/j.1365-313X.2008.03493.X

Schmid, M., Davison, T. S., Henz, S. R., Pape, U. J., Demar, M., Vingron, M., et al. (2005). A gene expression map of Arabidopsis thaliana development. Nat. Genet. 37, 501-506. doi: 10.1038/ng1543

Seo, P. J., Kim, S.-G., and Park, C.-M. (2008). Membrane-bound transcription factors in plants. Trends Plant Sci. 13, 550-556. doi: 10.1016/j.tplants.2008. 06.008

Shah, S. T., Pang, C., Fan, S., Song, M., Arain, S., and Yu, S. (2013). Isolation and expression profiling of GhNAC transcription factor genes in cotton (Gossypium hirsutum L.) during leaf senescence and in response to stresses. Gene 531, 220-234. doi: 10.1016/j.gene.2013.09.007

Shan, W., Kuang, J.-F., Chen, L., Xie, H., Peng, H.-H., Xiao, Y.-Y., et al. (2012). Molecular characterization of banana NAC transcription factors and their interactions with ethylene signalling component EIL during fruit ripening. J. Exp. Bot. 63, 5171-5187. doi: 10.1093/jxb/ers 178

Sierro, N., Battey, J. N., Ouadi, S., Bovet, L., Goepfert, S., Bakaher, N., et al. (2013). Reference genomes and transcriptomes of Nicotiana sylvestris and Nicotiana tomentosiformis. Genome Biol. 14:R60. doi: 10.1186/gb-2013-14-6-r60

Singh, A. K., Sharma, V., Pal, A. K., Acharya, V., and Ahuja, P. S. (2013). Genomewide organization and expression profiling of the NAC transcription factor family in potato (Solanum tuberosum L.). DNA Res. 20, 403-423. doi: 10.1093/ dnares/dst019

Takada, S., Hibara, K., Ishida, T., and Tasaka, M. (2001). The CUPSHAPED COTYLEDON1 gene of Arabidopsis regulates shoot apical meristem formation. Development 128, 1127-1135.

Takasaki, H., Maruyama, K., Takahashi, F., Fujita, M., Yoshida, T., Nakashima, K., et al. (2015). SNAC-As, stress-responsive NAC transcription factors, mediate ABA-inducible leaf senescence. Plant J. 84, 1114-1123. doi: 10.1111/tpj.13067

Tamura, K., Stecher, G., Peterson, D., Filipski, A., and Kumar, S. (2013). MEGA6: molecular evolutionary genetics analysis version 6.0. Mol. Biol. Evol. 30, 27252729. doi: 10.1093/molbev/mst197

Tranbarger, T. J., Fooyontphanich, K., Roongsattham, P., Pizot, M., Collin, M., Jantasuriyarat, C., et al. (2017). Transcriptome analysis of cell wall and NAC domain transcription factor genes during Elaeis guineensis fruit ripening: evidence for widespread conservation within monocot and eudicot lineages. Front. Plant Sci. 8:603. doi: 10.3389/fpls.2017.00603

Uauy, C., Distelfeld, A., Fahima, T., Blechl, A., and Dubcovsky, J. (2006). A NAC gene regulating senescence improves grain protein, zinc, and iron content in wheat. Science 314, 1298-1301. doi: 10.1126/science.1133649

van der Graaff, E., Schwacke, R., Schneider, A., Desimone, M., Flügge, U.-I., and Kunze, R. (2006). Transcription analysis of arabidopsis membrane transporters and hormone pathways during developmental and induced leaf senescence. Plant Physiol. 141, 776-792. doi: 10.1104/pp.106.079293
Vroemen, C. W., Mordhorst, A. P., Albrecht, C., Kwaaitaal, M. A. C. J., and de Vries, S. C. (2003). The CUP-SHAPED COTYLEDON3 gene is required for boundary and shoot meristem formation in Arabidopsis. Plant Cell 15, 1563-1577. doi: 10.1105/tpc.012203

Wang, N., Zheng, Y., Xin, H., Fang, L., and Li, S. (2013). Comprehensive analysis of NAC domain transcription factor gene family in Vitis vinifera. Plant Cell Rep. 32, 61-75. doi: 10.1007/s00299-012-1340-y

Wei, S., Gao, L., Zhang, Y., Zhang, F., Yang, X., and Huang, D. (2016). Genomewide investigation of the NAC transcription factor family in melon (Cucumis melo L.) and their expression analysis under salt stress. Plant Cell Rep. 35, 1827-1839. doi: 10.1007/s00299-016-1997-8

Wu, A., Allu, A. D., Garapati, P., Siddiqui, H., Dortay, H., Zanor, M.-I., et al. (2012). JUNGBRUNNEN1, a reactive oxygen species-responsive NAC transcription factor, regulates longevity in Arabidopsis. Plant Cell 24, 482-506. doi: 10.1105/ tpc.111.090894

Xie, Q., Guo, H.-S., Dallman, G., Fang, S., Weissman, A. M., and Chua, N.-H. (2002). SINAT5 promotes ubiquitin-related degradation of NAC1 to attenuate auxin signals. Nature 419, 167-170. doi: 10.1038/nature00998

Yamaguchi, M., Ohtani, M., Mitsuda, N., Kubo, M., Ohme-Takagi, M., Fukuda, H., et al. (2010). VND-INTERACTING2, a NAC domain transcription factor, negatively regulates xylem vessel formation in Arabidopsis. Plant Cell 22, 1249-1263. doi: 10.1105/tpc.108.064048

Yan, H., Zhang, A., Ye, Y., Xu, B., Chen, J., He, X., et al. (2017). Genomewide survey of switchgrass NACs family provides new insights into motif and structure arrangements and reveals stress-related and tissue-specific NACs. Sci. Rep. 7:3056. doi: 10.1038/s41598-017-03435-z

Yang, S.-D., Seo, P. J., Yoon, H.-K., and Park, C.-M. (2011). The Arabidopsis NAC transcription factor VNI2 integrates abscisic acid signals into leaf senescence via the COR/RD genes. Plant Cell 23, 2155-2168. doi: 10.1105/tpc.111.084913

Zhang, J., Huang, G. Q., Zou, D., Yan, J. Q., Li, Y., Hu, S., et al. (2018). The cotton (Gossypium hirsutum) NAC transcription factor (FSN1) as a positive regulator participates in controlling secondary cell wall biosynthesis and modification of fibers. New Phytol. 217, 625-640. doi: 10.1111/nph.14864

Zhang, K., and Gan, S.-S. (2012). An abscisic acid-AtNAP transcription factorSAG113 protein phosphatase $2 \mathrm{C}$ regulatory chain for controlling dehydration in senescing Arabidopsis leaves. Plant Physiol. 158, 961-969. doi: 10.1104/pp. 111.190876

Zhang, Y., Cao, Y., Shao, Q., Wang, L., Wang, H., Li, J., et al. (2012). Regulating effect of ZmNAP gene on anti-senescence and yield traits of maize. J. Henan Agric. Sci. 41, 19-24.

Zhong, R., Lee, C., Zhou, J., McCarthy, R. L., and Ye, Z.-H. (2008). A battery of transcription factors involved in the regulation of secondary cell wall biosynthesis in Arabidopsis. Plant Cell 20, 2763-2782. doi: 10.1105/tpc.108. 061325

Conflict of Interest Statement: The authors declare that the research was conducted in the absence of any commercial or financial relationships that could be construed as a potential conflict of interest.

Copyright (c) $2018 \mathrm{Li}$, Li, Chao, Zhang, Wang and Guo. This is an open-access article distributed under the terms of the Creative Commons Attribution License (CC BY). The use, distribution or reproduction in other forums is permitted, provided the original author(s) and the copyright owner(s) are credited and that the original publication in this journal is cited, in accordance with accepted academic practice. No use, distribution or reproduction is permitted which does not comply with these terms. 